\title{
ÉTUDE AU SPECTROMETRE DE MASSE DES PRODUITS D'ÉVAPORATION DU BIOXYDE DE TITANE ET DU TITANATE DE BARYUM
}

\author{
Par Guy MESNARD, Robert UZAN et Bernard CABAUD, \\ Laboratoire d'Électronique et de Physique du Solide, Faculté des Sciences de Lyon.
}

\begin{abstract}
Résumé. - Les produits étudiés $\left(\mathrm{Ti}, \mathrm{TiO}_{2}\right.$ et $\left.\mathrm{BaTiO}_{3}\right)$ sont chauffés sur support de tungstène dans la source d'ions du spectromètre. On a provoqué séparément l'ionisation par bombardement électronique et par effet Langmuir. Les courants ioniques obtenus sont précisés en fonction de la température et on analyse les mécanismes de leur obtention. On donne en outre les potentiels d'apparition de quelques ions.
\end{abstract}

Abstract. - The products studied $\left(\mathrm{Ti}, \mathrm{TiO}_{2}\right.$ and $\left.\mathrm{BaTiO}_{3}\right)$ are heated on a tungsten base in the ion source of the spectrometer.

Ionization has been produced separately by electron bombardment and by the Langmuir effect. The influence of temperature on the ion currents obtained is specified and the mechanisms of their production are analysed. Appearance potentials of some ions are also given.

En relation avec des travaux concernant la préparation de couches évaporées de titanate de baryum, il a paru intéressant d'étudier au spectrographe de masse les produits d'évaporation du titanate. Deux techniques ont été utilisées pour l'ionisation des vapeurs, le bombardement électronique et le contact avec un filament chaud (effet Langmuir). La première permettra de préciser les phénomènes par la détermination des potentiels d'apparition des ions, à l'aide de la méthode de l'extrapolation linéaire [1].

I. Technique expérimentale. $-a$. LA source D'Évaporation. - Le titanate de baryum a été préparé à partir de bioxyde de titane "Carlo Erba " et de carbonate de baryum Prolabo. On a aussi étudié le bioxyde de titane et le titane métallique. Les produits en poudre $\left(\mathrm{BaTiO}_{3}\right.$ ou $\left.\mathrm{TiO}_{2}\right)$, mis en suspension dans du collodion à l'acétate d'amyle, sont déposés sur une face d'un ruban de tungstène chauffant. Pour le titane, de petits cavaliers sont posés sur le ruban ; l'ensemble est ensuite porté à haute température dans une installation de vide annexe, afin d'obtenir un revêtement de titane aussi uniforme que possible.

$b$. Détermination De la température De L'ÉLÉMENT CHAUFfant. - Nous avons utilisé deux techniques différentes :

1) Un thermocouple tungstène rhénié $26 \%-5 \%$ est soudé électriquement sur la face nue du ruban de tungstène. Cette méthode présente l'inconvénient de provoquer une contamination par le rhénium et de créer une zone froide au milieu du ruban, malgré le faible diamètre des fils de couples utilisés $(0,05 \mathrm{~mm})$.

2) Un petit écran de tantale est placé derrière le ruban chauffant, aussi près que possible de celui-ci et dans une position bien déterminée. Il prend une température inférieure à celle du tungstène ; on lui soude les fils d'un couple platine-platine rhodié (diamètre $0,05 \mathrm{~mm}$ ).

L'étalonnage de la température de l'élément chauffant en fonction de celle de l'écran est effectué à l'aide d'un micropyromètre optique Ribaud, une fois l'équilibre de température atteint. Une bonne reproductibilité a été obtenue. Un nouvel étalonnage était effectué à chaque changement de filament.

c. Le spectrographe. - L'appareil (Atlas Werke CH3 modifié) est à simple focalisation et à secteur magnétique de $60^{\circ}$. La même source permet l'ionisation soit par un faisceau électronique de $40 \mu \mathrm{A}$, soit par un ruban, parallèle à la fente extractrice, porté à une température fixe (2 $\left.400^{\circ} \mathrm{K}\right)$, toujours supérieure à celle de la source d'évaporation [2]. La sensibilité du collecteur d'ions est de quelques $10^{-15}$ ampère.

II. Résultats expérimentaux : Source à faisceau d'électrons. - Nous présenterons les évolutions des courants ioniques correspondant aux diverses masses " apparentes " par $\log I\left(\mathrm{X}^{+}\right)$en fonction de $1 / T\left({ }^{\circ} \mathrm{K}\right)$. Les courbes sont sensiblement rectilignes, en accord avec les lois de la vaporisation, bien que l'on soit en régime dynamique et que dans une certaine mesure on ait affaire à une évaporation. Nous donnerons en outre les valeurs des différents potentiels d'apparition.

a. Titane métallique. - On a observé les ions $\mathrm{Ti}^{+}, \mathrm{Ti}^{++}$et $\mathrm{Ti}^{+++}$. Avec la représentation adoptée, la figure 1 montre que l'on obtient des 
droites parallèles de pente $5 \times 10^{4}$. Pour une température donnée les intensités relatives sont: 100 pour $\mathrm{Ti}^{+}, 35$ pour $\mathrm{Ti}^{++}$et 2 pour $\mathrm{Ti}^{+++}$. Ces ions sont tous produits par les impacts électroniques sur la vapeur de titane, les valeurs des sections efficaces étant entre elles comme les nombres 100 , 35,2 , pour la tension d'accélération de 70 volts.

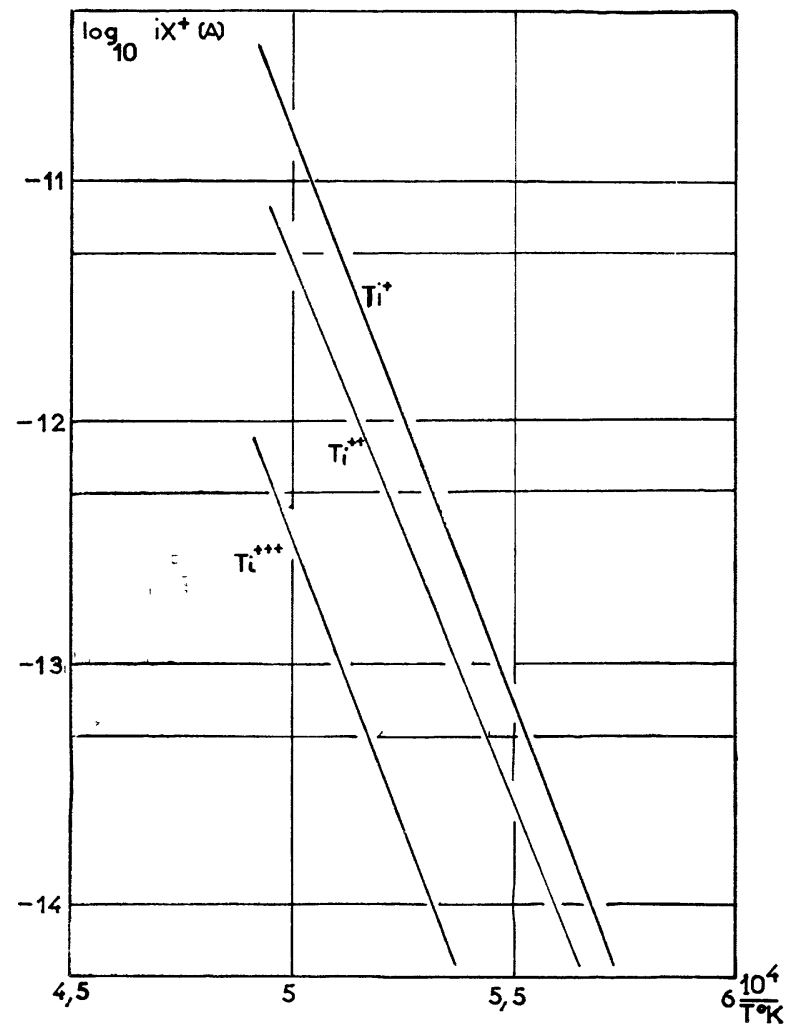

FIG. 1.

Nous avons obtenu, en adoptant l'argon comme étalon, les potentiels d'apparition suivants :

- pour $\mathrm{Ti}^{+}, 6,6 \pm 0,2 \mathrm{~V}$ (en accord avec la valeur spectroscopique $6,8 \mathrm{~V}$ ) ;

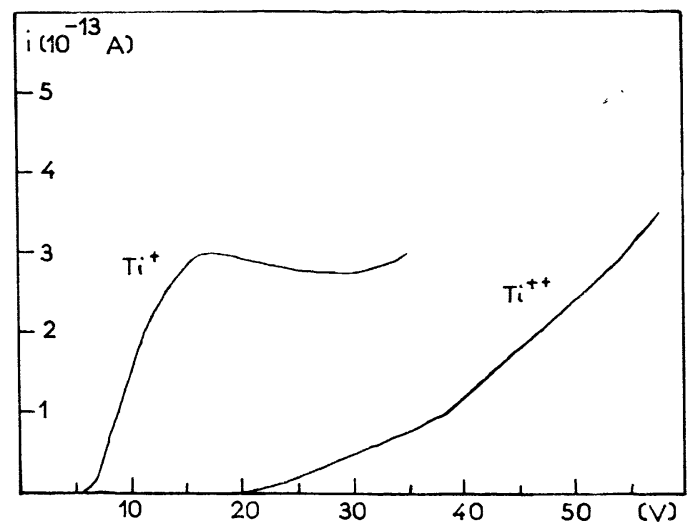

FIG. 2.
- pour $\mathrm{Ti}^{++}, 21,5 \pm 1 \mathrm{~V}$ (valeur spectroscopique $20,4 \mathrm{~V}$ ) ; on observe en outre un coude vers 38 volts $($ fig. 2$)$;

- pour $\mathrm{Ti}^{+++}, 50 \pm 1 \mathrm{~V}$ (valeur spectroscopique $48 \mathrm{~V}$ ).

$b$. Bioxyde de titane. - Les ions obtenus sont $\mathrm{TiO}_{2}^{+}, \mathrm{TiO}_{2}^{++}, \mathrm{TiO}^{+}, \mathrm{TiO}^{++}, \mathrm{Ti}^{+}, \mathrm{Ti}^{++}, \mathrm{O}_{2}^{+}$et $\mathrm{O}^{+}$.La figure 3 représente des courbes de $\log I\left(\mathrm{X}^{+}\right)$ en fonction de $1 / T$.

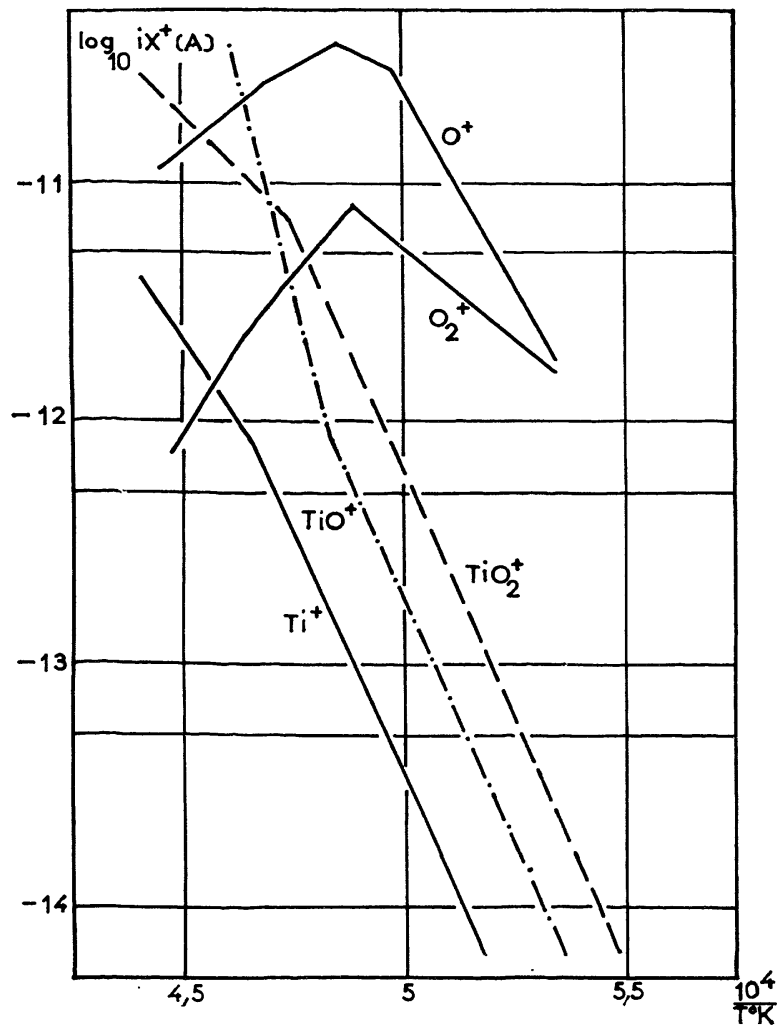

Fig. 3.

1. Ions $\mathrm{O}^{+}$et $\mathrm{O}_{2}^{+}$. - Malgré une certaine imprécision due au "résiduel ", la présence de ces ions montre que $\mathrm{TiO}_{2}$ est décomposé partiellement avec libération d'oxygène moléculaire et atomique. La croissance initiale en fonction de la température est liée à l'évaporation croissante de $\mathrm{TiO}_{2}$; la décroissance observée au delà de $2050^{\circ} \mathrm{K}$ environ montre que les ions oxygène ne peuvent être produits que pour une part par l'impact électronique sur les molécules de $\mathrm{TiO}_{2}$ dont la concentration continue à augmenter.

Une importante dissociation thermique de $\mathrm{TiO}_{2}$ est donc obtenue ; elle serait provoquée essentiellement par la présence du ruban chauffant. La décroissance peut s'expliquer par l'oxydation du tungstène et par l'adsorption de l'oxygène sur le titane qui s'évapore également et se dépose sur les 
parois (effet " getter »). On constate également que le rapport $I\left(\mathrm{O}^{+}\right) / I\left(\mathrm{O}_{2}^{+}\right)$augmente avec la température, ce que l'on peut considérer comme normal.

2. Ions contenant du titane. - La présence de la vapeur de $\mathrm{TiO}_{2}$ est décelée à partir de $1850^{\circ} \mathrm{K}$ environ. Les autres courants ioniques, plus faibles, varient d'abord sensiblement de la même facon en fonction de la température : la pente des droites représentatives est de $4 \times 10^{4}$. Vers $2075^{\circ} \mathrm{K}$, la pente augmente pour $\mathrm{TiO}^{+}$(elle devient égale à $6,5 \times 10^{4}$ ), alors qu'elle diminue pour $\mathrm{TiO}_{2}^{+}$et $\mathrm{Ti}^{+}$. L'augmentation de pente relative à $\mathrm{TiO}^{+}$montre qu'il existe deux processus de production de TiO. Nous avons déjà envisagé la dissociation thermique de $\mathrm{TiO}_{2}$; il faut y ajouter la réduction par le tungstène, qui est importante aux températures élevées. A ces températures en effet l'obtention de TiO ne s'accompagne plus d'une production d'oxygène sensible; en outre on a noté la diminution de la pente de la courbe de $\mathrm{TiO}_{2}^{+}$; si on considère l'ensemble $\mathrm{TiO}_{2}+\mathrm{TiO}$, une loi exponentielle simple est vérifiée dans toute la gamme des températures.

Les courbes de sections efficaces des ions sont reproduites sur la figure 4 . On a obtenu les potentiels d'apparition suivants :

- pour $\mathrm{TiO}_{2}^{+}, 11 \pm 0,3 \mathrm{~V}$;

- pour $\mathrm{TiO}^{+}, 5,5 \pm 0,5 \mathrm{~V}$ et $8 \pm 0,5 \mathrm{~V}$;

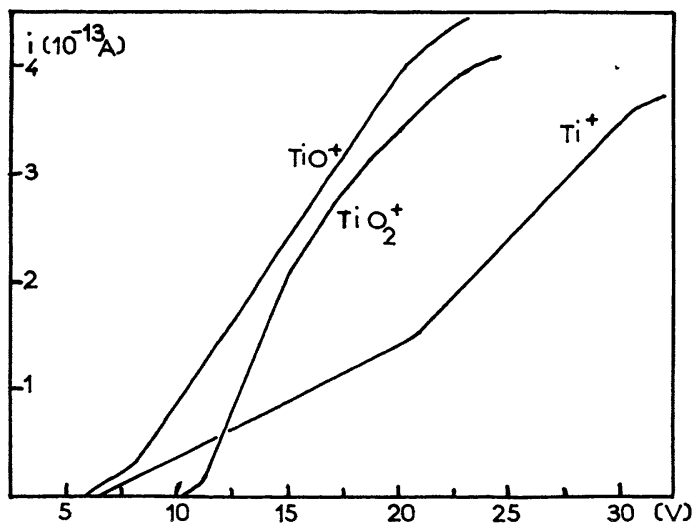

FIG. 4.

Pour $\mathrm{Ti}^{+}$la courbe de section efficace diffère de celle de la figure 2. Cet ion ne provient donc pas uniquement de l'ionisation de vapeur de titane par l'impact électronique : le premier potentiel d'apparition $(6,3 \pm 0,5 \mathrm{~V})$ correspond bien à la vapeur de titane, un deuxième $(21 \pm 1 \mathrm{~V})$ serait dû à l'impact soit sur $\mathrm{TiO}_{2}$ soit sur $\mathrm{TiO}$, la première hypothèse étant la plus plausible d'après les formes comparées des courbes.

La présence de titane métallique s'explique évidemment par la dissociation et la réduction de $\mathrm{TiO}_{2}$, la proportion en étant d'ailleurs faible.

c. Titanate de baryum. - 1. Évolution des pics avec la température. - Les résultats expérimentaux sont représentés sur la figure 5 .

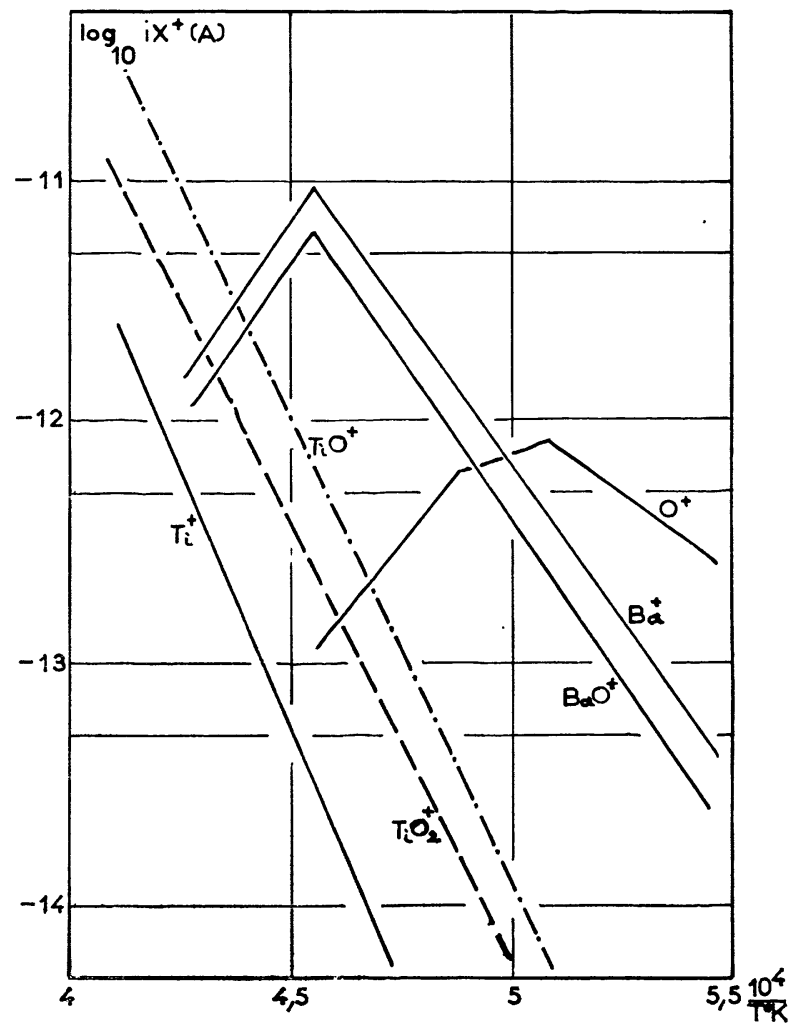

FIG. 5 .

Les ions $\mathrm{Ba}^{+}, \mathrm{BaO}^{+}$et $\mathrm{O}^{+}$apparaissent les premiers lorsqu'on élève la température. Leur présence montre qu'une décomposition du titanate a lieu. On n'obtiendra d'ailleurs jamais de pic correspondant à $\mathrm{BaTiO}_{3}$.

L'évaporation de $\mathrm{BaO}$ se manifeste toutefois à des températures plus élevées que si l'on part de l'oxyde lui-même [1]. Comme dans l'étude de $\mathrm{BaO}$ sur tungstène, les pics $\mathrm{BaO}^{+}$et $\mathrm{Ba}^{+}$sont comparables en amplitude : $\mathrm{Ba}^{+}$est produit pour une part par impact électronique sur $\mathrm{BaO}$, ce qui explique la présence d'oxygène $\mathrm{O}^{+}$; on n'obtient pas d'ions $\mathrm{O}_{2}^{+}$.

Les ions $\mathrm{TiO}_{2}^{+}, \mathrm{TiO}^{+}$et $\mathrm{Ti}^{+}$n'apparaissent eux mêmes qu'à des températures plus élevées que lors de l'étude de $\mathrm{TiO}_{2}$. Aux basses températures la décomposition de $\mathrm{BaTiO}_{3}$ est donc limitée; elle augmente ensuite avec celle-ci. Dans les premiers stades de cette décomposition, il se formerait du titanate plus riche en titane, tel que $\mathrm{BaTi}_{2} \mathrm{O}_{5}$; en effet l'évaporation de $\mathrm{TiO}_{2}$ est très faible.

Aux températures élevées les ions $\mathrm{BaO}^{+}, \mathrm{Ba}^{+}$ et $\mathrm{O}^{+}$deviennent moins abondants, alors que les courants ioniques $\mathrm{TiO}_{2}^{+}, \mathrm{TiO}^{+}$et $\mathrm{Ti}^{+}$continuent à 
augmenter avec la température (aucun phénomène d'épuisement n'est observé jusqu'à $2400^{\circ} \mathrm{K}$ ) ; il est raisonnable d'admettre la formation de tungstate de baryum.

Les courants ioniques pour $\mathrm{TiO}^{+}, \mathrm{TiO}_{2}^{+}$et $\mathrm{Ti}^{+}$ sont entre eux comme 100,35 et 5 : on remarque que le pic le plus important est $\mathrm{TiO}^{+}$comme dans l'étude précédente $b$ dans le même domaine de température.

La pente des droites $\left(\mathrm{BaO}^{+}\right)$et $\left(\mathrm{Ba}^{+}\right)$, égale à $2,8 \times 10^{4}$, est voisine de celles qui ont été obtenues antérieurement [1]. Fn ce qui concerne les ions contenant du titane, la pente est voisine de $4 \times 10^{4}$, comme précédemment aux basses températures.

2. Potentiels d'apparition. - Nous avons consigné dans le tableau I les valeurs moyennes obtenues pour les différents ions. Les deux valeurs obtenues pour $\mathrm{Ba}^{+}$correspondent aux deux origines de ces ions : présence de vapeur de baryum et dissociation de $\mathrm{BaO}$ par impact électronique. Ces résultats sont en accord avec des mesures antérieures [1]. En particulier on retrouve une faible valeur du p. a. de $\mathrm{Ba}^{+}$et on obtient en même temps des ions spontanés.

\section{TABLEAU I}

$$
\begin{aligned}
& \mathrm{Ba}^{+} \\
& \mathrm{BaO}^{+} \\
& \mathrm{TiO}^{+} \\
& \mathrm{TiO}_{2}^{+} \\
& \mathrm{Ti}^{+} \\
& \begin{array}{r}
4 \\
5,2 \pm 0,2 \mathrm{~V} \\
5,5 \pm 0,5 \mathrm{~V} \\
9 \pm 0,2 \mathrm{~V} \\
11,5 \pm 0,2 \mathrm{~V}
\end{array} \\
& 13,7 \pm 0,5 \mathrm{~V} \\
& 8 \pm 0,5 \mathrm{~V} \\
& 20 \pm 0,2 \mathrm{~V}
\end{aligned}
$$

En ce qui concerne $\mathrm{TiO}^{+}$, le p. a. de $5,5 \mathrm{~V}$ déjà obtenu dans l'étude de $\mathrm{TiO}_{2}$, correspond à l'ionisation directe par impact électronique. Pour $\mathrm{TiO}_{2}^{+}$ ( fig. 6), le p. a. est inférieur à celui de l'étude (II, b). Pour $\mathrm{Ti}^{+}$, le premier p. a. est beaucoup plus élevé et n'avait pas été observé précédemment ; cet ion ne provient pas de vapeur de titane, qui n'existerait pas, mais est produit par impact électronique sur les oxydes de titane. L'absence de vapeur de titane

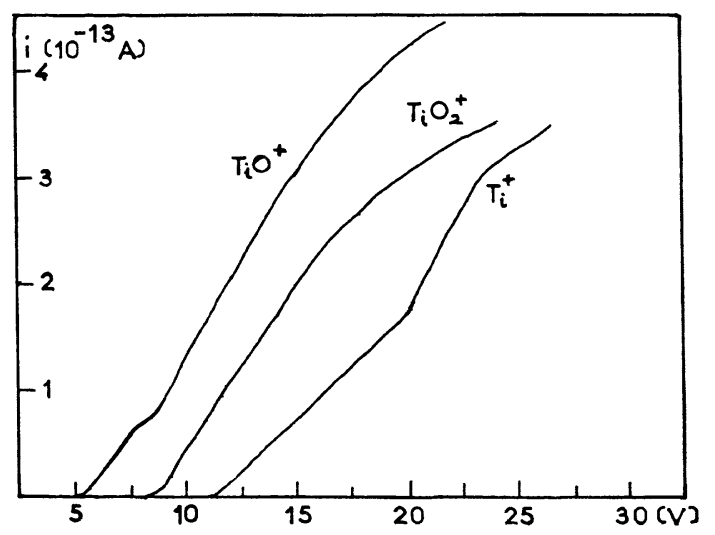

FIG. 6 . résulterait du recouvrement du tungstène par une pellicule de tungstate diminuant son rôle réducteur.

III. Résultats expérimentaux : Source thermoionique. - On observe ici les ions émis directement ou produits au contact du ruban d'ionisation thermique.

a. Titane métallique. - On observe l'ion $\mathrm{Ti}^{+}$à partir de $1750^{\circ} \mathrm{K}$; l'amplitude du pic augmente régulièrement jusqu'à $2100^{\circ} \mathrm{K}$, température finale de l'étude.

$b$. Bioxyde de titane. - Les seuls ions observé sont $\mathrm{Ti}^{+}$et $\mathrm{TiO}^{+}$. Nous n'avons pas obtenu d'ions $\mathrm{TiO}_{2}^{+}$, ni O${ }_{2}^{+}, \mathrm{ni} \mathrm{O} \mathrm{O}^{+}$.

L'absence des ions $\mathrm{TiO}_{2}^{+}$montre que cette molécule s'ionise difficilement, car son existence a été mise en évidence en (II, b). Par contre Ti et TiO sont facilement ionisés et le comportement général est en accord avec (II, $b$ ).

La figure 7 représente les résultats en fonction de la température. Pour $\mathrm{TiO}^{+}$on obtient dans la représentation adoptée deux demi-droites de pentes $4 \times 10^{4}$ et $6,6 \times 10^{4}$, en très bon accord avec (II, $\left.b\right)$. Le pic $\mathrm{Ti}^{+}$est relativement plus faible ici, bien que le taux d'ionisation soit au moins aussi élevé que celui de $\mathrm{TiO}$; ceci serait dû au fait que le fais-

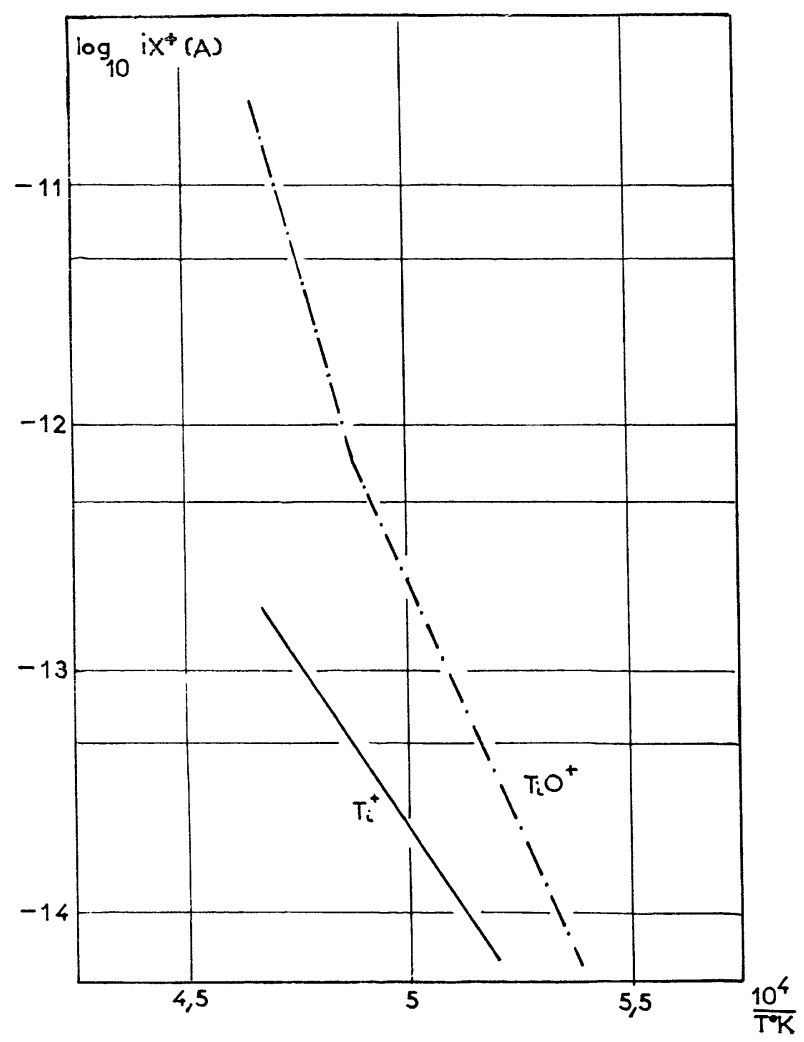

FIG. 7. 
ceau électronique créait aussi des ions par dissociation. $\mathrm{L}^{\prime}$ absence de pics $\mathrm{O}_{2}^{+}$et $\mathrm{O}^{+}$tient à la très faible ionisation de l'oxygène par contact avec un solide chaud.

$c$. Titanate de baryum. - Les ions essentiels sont $\mathrm{Ba}^{+}$et $\mathrm{BaO}^{+}$. Le rapport $I\left(\mathrm{Ba}^{+}\right) I I\left(\mathrm{BaO}^{+}\right)$est de l'ordre de 1000 : l'ionisation de $\mathrm{BaO}$ est peu accusée, alors qu'elle est importante pour $\mathrm{Ba}$, le pic $\mathrm{Ba}^{+}$étant du même ordre de grandeur qu'en (II, c) malgré l'absence des ions $\mathrm{Ba}^{+}$dus à la dissociation de $\mathrm{BaO}$ par impact électronique. L'absence d'ions $\mathrm{O}^{+}$est normale, mais la formation d'ions négatifs $\mathrm{O}^{-} \mathrm{n}^{\text {'est }}$ pas exclue.

L'absence d'ions $\mathrm{TiO}_{2}^{+}$est normale d'après les considérations énoncées en (III, b), mais l'évaporation de $\mathrm{TiO}_{2}$ a été prouvée en (II, $c$ ). L'évaporation de TiO a aussi été prouvée ; or on n'a obtenu ici que des traces de $\mathrm{TiO}^{+}$et $\mathrm{Ti}^{+}$. Les molécules de $\mathrm{TiO}$ s'ioniseraient mal ici, contrairement au cas de l'étude (III, $b$ ), car le ruban chaud ionisant serait recouvert de tungstate sous l'action des vapeurs de $\mathrm{Ba}, \mathrm{BaO}$ et oxygène. L'absence de $\mathrm{Ti}^{+}$est normale : on a vu en (II, c) que cet ion était obtenu uniquement par bombardement.

Conclusion. - Ce travail, qui avait pour but essentiel, la détermination des produits d'évaporation du titanate de baryum, a montré notamment :

1) que le titanate est décomposé avant toute évaporation appréciable ;

2) que le tungstène réagit lui-même sur le titanate ;

3) que les produits évaporés sont essentiellement $\mathrm{Ba}$ et $\mathrm{BaO}$ d'une part, $\mathrm{TiO}$ et $\mathrm{TiO}_{2}$ d'autre part ;

4) qu'il y a formation d'autres titanates et de tungstate de baryum.

A l'occasion de cette étude, nous avons déterminé quelques valeurs approximatives de potentiels d'apparition.

Manuscrit reçu le 20 octobre 1965.

\section{RÉFÉRENCES}

[1] DéJardin (G.), Mesnard (G.), Uzan (R.) et Cabaud (B.), C. R. Acad. Sc., 1962, 255, 1712-1714.

Mesnard (G.), Uzan (R.) et Cabaud (B.), Cahiers de Physique, 1963, 156, 333-343.
Cabaud (B.), Thèse de spécialité, Lyon, 1963.

[2] Inghram (M. G.) et Chupka (W. A.), Rev. Sc. Instr., $1953,24,518$. 